\title{
The development of the Heliometer of the Observatório Nacional
}

\author{
Victor d'Ávila ${ }^{1,2}$, Eugênio Reis ${ }^{1}$, Jucira Penna $^{1}$, Luiz Carlos \\ Oliveira $^{1,3}$, Alissandro Coletti ${ }^{4,5}$, Victor Matias, ${ }^{1,3}$ Alexandre \\ Andrei ${ }^{1,6}$ and Sergio Boscardin ${ }^{1}$ \\ ${ }^{1}$ Observatório Nacional/MCT, R. Gal. Jose Cristino 77, Rio de Janeiro, Brasil \\ email: victor@on.br \\ ${ }^{2}$ Uerj, ${ }^{3}$ NGC-51, ${ }^{4}$ Azeheb, ${ }^{5}$ FACC, ${ }^{6}$ OV $/$ UFRJ
}

\begin{abstract}
The Observatório Nacional at Rio de Janeiro has developed a new heliometer to perform accurate measurements of the solar diameter. The equipment makes use of split parabolic mirrors, rather than split achromatic lenses as objective. In its first campaign this reflector heliometer generated a few thousands of images of the Sun through which we evaluate the precision of the solar diameter measurements as been 5 mas. The mechanical and thermal stability of the instrument is guaranteed by the use of a telescope tube manufactured in carbon fiber. The tube firmly holds the heliometric mirrors and the CCD camera that collects the images. The perfect lodging between the two half-mirrors over its supporting plate defines the angular instrumental separation between the two images of the sun. The heliometric mirrors and supporting plate have been made in ceramic material in order to ensure the stability of the optical configuration and to maintain the two images of the solar disk displaced by a fixed angle in relation to each other. To verify the stability of the instrument we designed the procedures of collimation and comparison. The collimation consists of using the heliometer for measuring the diameter of an artificial solar image specifically created for this purpose. The comparison is performed by measuring the residual unfold of a punctual image after been unfold by two opposed heliometers. The heliometric approach allows the measurement of the solar diameter at any heliolatitude and can be undertaken as often as desired. Computer programs have been developed for automated image acquisition and analysis. The optical and mechanical design has been performed by using 3D computer aided design software.
\end{abstract}

Keywords. Sun: fundamental parameters; instrumentation: heliometers; techniques: photometric

\section{Introduction}

In order to follow up and improve on the transit-time, Astrolabe CCD surveying of the solar at diameter at Observatório Nacional/MCT, a second approach was develloped, which consists of the direct astrometric measurement of the figure of the solar disk with a heliometer.

The fundamental difficulty of measuring a small angle by optical means comes from the fact that the measurement is directly dependent on the positioning of the focal plane of the instrument. In fact, the difficulty pointed out here for performing angular measurements is also present in reflecting telescopes. The solution presented in this paper consists of a using bisected mirror rather than achromatic doublet as has been adopted in the past (Geyer, 1984). Each half-mirror is tilted but an angle of 0.125 degrees in order to displace the images relatively to each other by one solar diameter approximately. In this way we will have opposite limbs of the sun almost in tangency in the focal plane 


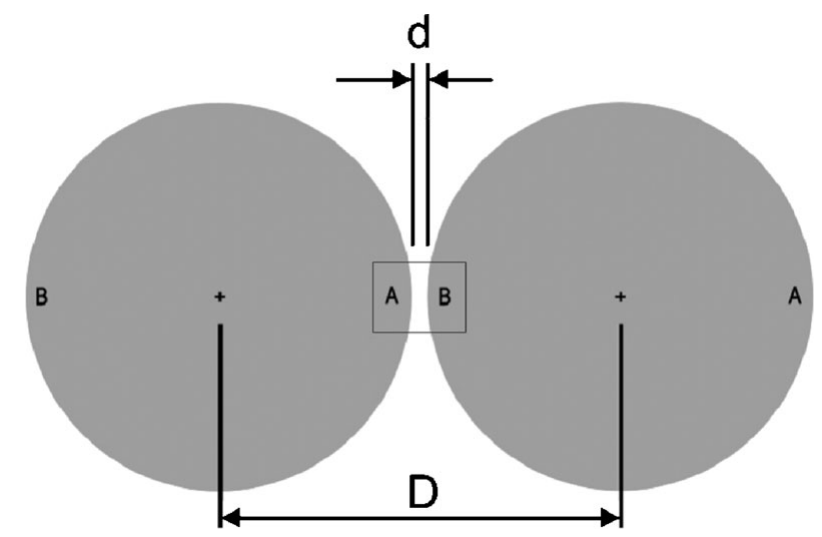

Figure 1. Schematic heliometric measurement: solar diameter $=\mathrm{D}-\mathrm{d}$.

(Fig. 1). With this setup we eliminate all chromatic defects, and also take advantage of the mechanical stability of mirrors.

\section{Heliometric Concept}

The basic idea behind the heliometric technique consists of doubling, in some way, the field of view of a telescope and applying a relative displacement between them, in such way to bring two nearby objects into close proximity. This approach is very useful as a way to reduce the measurement of finite angle, say of a few degrees, into something more convenient, and as small as 1 minute of arc, or so. Supposing that, in principle, the errors are proportional to the amount to be measured, we see that it is possible to get, a considerable increase in the measurements.

Another advantage of the method heliometric comes from the fact that we can perform measurements along any chosen heliolatitude and whenever desired. Of course we have to take into account the effects of the differential refraction between the limbs along different heliolatitudes. In order to have the observations made under similar conditions we have chosen the instrumental displacement of the images in such a way that the minimum distance between the limbs be approximately equal to the variation of the apparent solar diameter between aphelion and perihelion (Fig. 2).

In the first two years of the heliometer project we have built some prototypes to test different image duplication techniques:

(a) Two plane mirrors in angle positioned in front of the objective have been tried. That would offer the freedom to use a standard telescope to perform the task. It would imply, however, in more parts involved.

(b) The microscope prism approach also allows the duplication of the images. But that does not help in the dependency of the measurements with the focal distance.

(c) We also have made some experiments with the classical solution of bisecting refracting objectives. However, in general, objective lenses are chromatic doublet or even panchromatic triplet. It is very difficult to have all parts of one such objective maintained fixed in relation to each other. And we still have take into consideration chromatic effects of second order.

(d) Finally, we have worked with an entirely new approach, which consists of bisecting a primary mirror of a telescope. Now, two possibilities are presented and have been tried: The linear heliometer (in which we produce the displacement of the images by translating the two half-mirrors along the line of cut), and the angular heliometer (in 


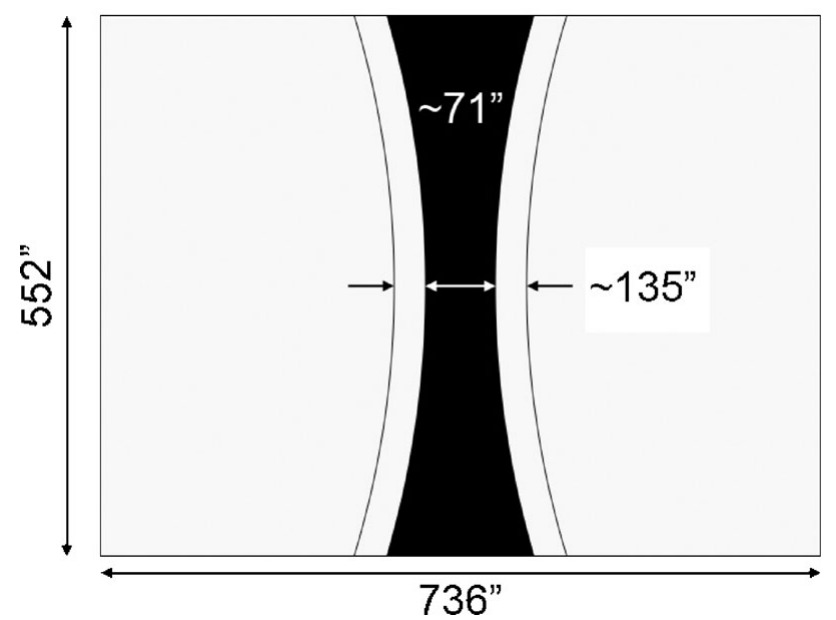

Figure 2. Aphelion and perihelion heliometric configuration.

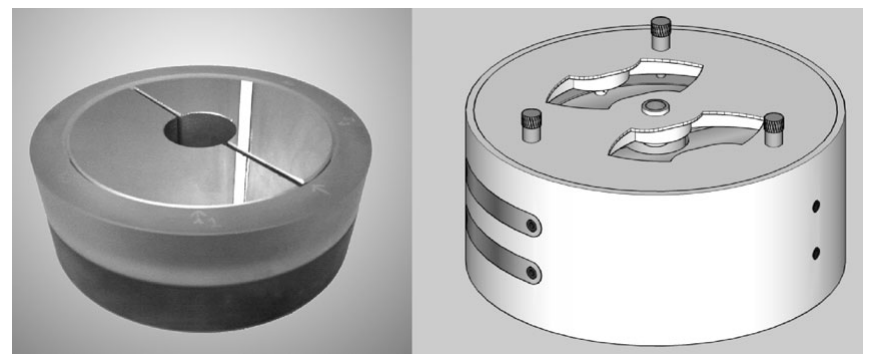

Figure 3. The actual heliometric mirror assembling and the project of its cell.

which we produce the displacement of the images by rotating the two half-mirrors along a line perpendicular to the line of cut).

The latter method was the choice for the construction of the Heliometer of the Observaório Nacional.

The heliometric mirror is all made of CCZ-HS, a ceramic material with very low thermal expansion coefficient $\left(0.0 \pm 0.2 \times 10^{-7} /{ }^{\circ} \mathrm{C}\right)$. The two half mirrors are immobilized, in relation to each other, by means of a external ring, all resting over an optical plate (Fig. 3a). Its cell guarantees the mechanical and geometrical stability for the entire set (Fig. 3b). The mask seen at the top of the cell has been designed to keep the two halfmirrors blocked in place and also assures that entrance pupil has a symmetric shape. The surface quality of the optical plate and the mirrors is better than $\lambda / 12$ and $\lambda / 20$, respectively.

The tube of the telescope is made of carbon fiber. This material, as well as extremely rigid, has very low coefficient of thermal expansion. It is mounted inside a stainless steel truss support and can rotate around its axis (Fig. 4).

In order to eliminate the secondary mirror the CCD chip was removed apart from the camera electronics and installed directly in the focal plane.

During the collimation and comparison procedures we observe the images of an artificial sun, which consists of a small ceramic disk placed in the optical axis of the instrument and immediately above the heliometric mirrors. A pair of thin nylon wires, in form of cross, have been installed over this artificial sun in order to provide a reference frame. 


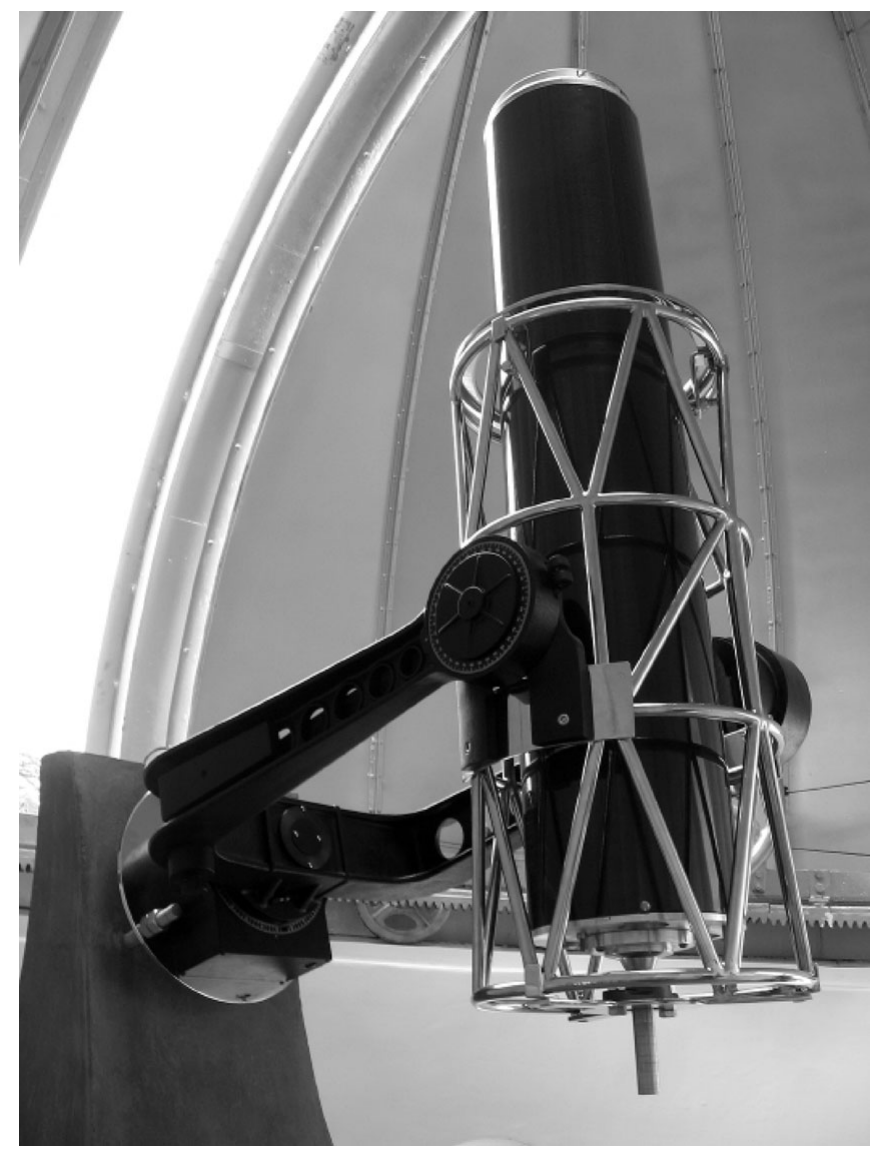

Figure 4. The Heliometer of the Observatório Nacional.

To perform the collimation procedure we install a parabolic mirror in front of the heliometer in such way to produce an image of the artificial sun at the infinite. During the collimation we observe the images of the two opposed limbs of the artificial sun in a way that is substantially similar to the real observation of the sun as can seen in Fig. 5a.

With this setup we are able to measure the displacement in two orthogonal direction. 1)- The angular displacement along the direction parallel to the cut is determined by the horizontal gap between the limbs. 2)- The angular displacement along the direction orthogonal to the cut can be evaluated by the vertical displacement of the images of the horizontal wire.

The comparison procedure, in its turn, is made by replacing parabolic mirror of the collimation by a secondary heliometer. This secondary heliometer produces an unfolded image of the artificial sun at the infinite. The primary heliometer then bring back these unfolded images to close proximity. In this configuration we observe the final images of nylon cross after been successively unfolded by the two opposing heliometers. We are then able to evaluate the remaining displacement along the directions of the two orthogonal wires (Fig. 5b). 

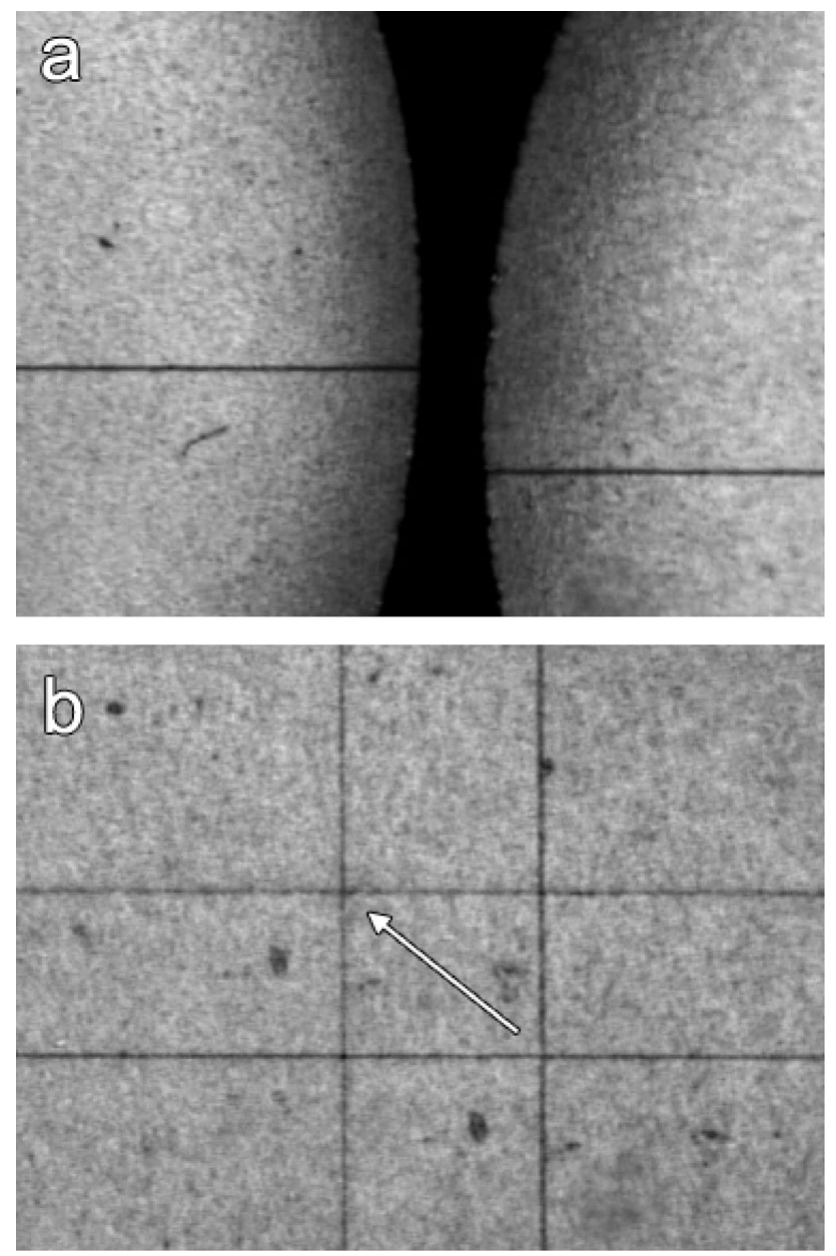

Figure 5. Collimation (a) and comparison (b) procedure images. The arrow indicates the displacement of the nylon cross center.

\section{Results}

In May 2009 there was an observational campaign that extended for 9 days, with over 70,000 heliometric images of the Sun. The heliometer exhibited a precision around 0.5 arc seconds for an isolated observation. And, we also see that in only one day of observations we can reach a precision about 5mas. The results are shown in Table 1.

These preliminary observations show a internal dispersion that is consistent with what should be expected to be due to the atmospheric seeing alone.

The results show that the values of the measured semi-diameters follow a normal distribution what indicates the absence of significant instrumental errors (Fig. 6). As the observational procedure is able to capture and process thousands of heliometric images per day, the measurements can achieve a significantly high accuracy in a short period of time. 
Table 1. First heliometric measurements of the solar semi-diameter.

\begin{tabular}{ccc}
\hline Day $^{1}$ & SD $^{2}(")$ & $\sigma(")$ \\
\hline 13 & $959.084 \pm 0.005$ & 0.461 \\
14 & $959.656 \pm 0.006$ & $\mathbf{0 . 5 5 9}$ \\
18 & $959.440 \pm 0.006$ & $\mathbf{0 . 5 3 3}$ \\
19 & $959.306 \pm 0.005$ & 0.459 \\
20 & $959.367 \pm 0.006$ & $\mathbf{0 . 5 2 1}$ \\
21 & $959.503 \pm 0.007$ & $\mathbf{0 . 6 3 9}$ \\
25 & $959.646 \pm 0.006$ & 0.515 \\
26 & $959.811 \pm 0.006$ & 0.501 \\
27 & $959.823 \pm 0.006$ & 0.528 \\
\hline & $959.519 \pm 0.002$ & 0.566
\end{tabular}

Notes:

${ }^{1}$ May 2009.

${ }^{2}$ Solar semi-diameter at $1 \mathrm{AU}$.

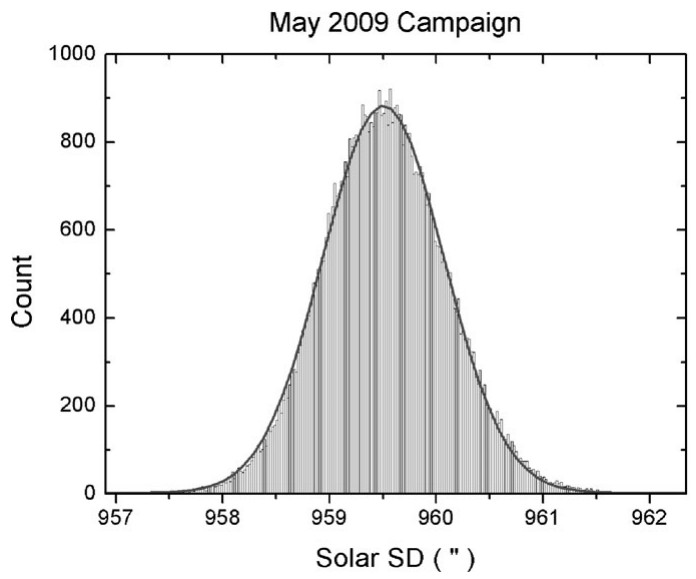

Figure 6. Solar semi-diameter distribution with a gaussian fit.

\section{Conclusion}

The materials that form the Heliometer have thermal and mechanical stability to dex-7. The number of optical parts is minimized and their quality is better than $\lambda / 12$. Original software for the automated collection and analysis of the images was developed. All along the project was developed by actual implements on to a trial heliometer. By using the final version fully realized, we conducted an observational campaign through 9 days, deriving more than 70,000 heliometric images of the Sun These results indicate a precision of 0.5 arcseconds, with no instrumental bias, and limited only by the provisional atmospheric modeling. Therefore, as meant from start, an accuracy of 5 mas can be achieved. The first results indicate that we should pursue this approach and initiate a campaign of observations aiming to cover this solar cycle which is just in its beginning, at least.

\section{Acknowledgements}

The authors acknowledge partial financial support from the Brazilian Science Funding Agencies FAPERJ, CNPq, CAPES and FINEP. We also acknowledge the NGC-51 group.

\section{Reference}

Geyer, E. H. 1984, Ap\&SS, 110, 183 